\title{
Regenerated Cellulose by the Lyocell Process, a Brief Review of the Process and Properties
}

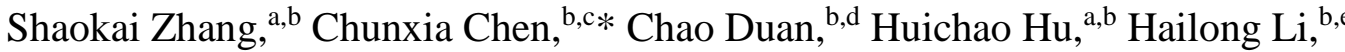

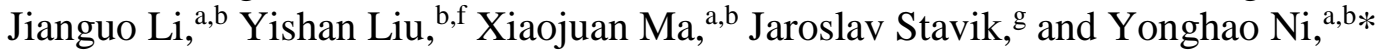 \\ Lyocell fiber has emerged as an important class of regenerated cellulose \\ that is produced based on the $\mathrm{N}$-methyl morpholine-N-oxide (NMMO) \\ dissolution method, and it has unique properties compared to viscose \\ fiber. The NMMO technology provides a simple, resource-conserving, and \\ environmentally friendly method for producing regenerated cellulose fiber. \\ In this paper, the manufacturing process, environmental impact, and \\ product quality of lyocell fiber are reviewed and compared with those of \\ the conventional viscose fiber.
}

Keywords: Lyocell fibers; NMMO; Manufacturing process; Environmental impact; Viscose fibers

Contact information: a: College of Material Engineering, Fujian Agriculture and Forestry University, Fuzhou 350108, China; b: Limerick Pulp and Paper Centre and Department of Chemical Engineering, University of New Brunswick, Fredericton, New Brunswick E3B 5A3, Canada; c: Dongguan Quality Supervision Testing Center (National Paper Products Quality Supervision Inspection Center), Dongguan 523808, China; d: College of Bioresources Chemical and Materials Engineering, Shaanxi University of Science and Technology, Xi'an 710021, China; e: Tianjin Key Laboratory of Pulp and Paper, Tianjin University of Science and Technology, Tianjin 300457, China; f: Sichuan Business and Technology College, Dujiangyan 611830, China; g: Schweighofer Fiber GmbH, Salzachtalstraße 88, Postfach 62, 5400 Hallein, Austria;*Corresponding authors:yonghao@unb.ca jane01212000@126.com

\section{INTRODUCTION}

The textile industry not only provides one of the necessities of life, but it also plays a very important role through its contribution to industrial output and employment opportunity, which is particularly true in Asia. In recent years, the demand for regenerated cellulose, which is characterized as sustainable material that is compatibility with the environment, in addition to having unique favorable properties, has experienced rapid growth (Quintana et al. 2013; Miao et al. 2014; Tian et al. 2014; Duan et al. 2015; Chen et al. 2015; Wang et al. 2006, 2015). The global production of textile fibers was predicted to increase from 72.5 million tons in 2010 to 133.5 million tons in 2030 (Haemmerle 2011). On the other hand, the cotton production will have a ceiling of 26 to 28 million tons per year (Sixta et al. 2013). For this reason, it is expected that the global dissolving pulp production will continue to grow (Chen et al. 2015; Li et al. 2015; Duan et al. 2016).

For many years, the viscose process has been the most dominant process for the production of regenerated cellulose fibers, while others such as the lyocell process, and the Cupro process, are rather limited (Hauru et al. 2014). In the conventional viscose process, the use of carbon disulfide, an environmentally sensitive chemical is one of the disadvantages. The development of carbon disulfide free- dissolving and regenerating cellulose, therefore, has been of practical interests.

$\mathrm{N}$-methyl morpholine-N-oxide (NMMO), as a replacement for carbon disulfide, was developed as a solvent for cellulose by Eastman Kodak Inc. (Blackburn 2005; Sinclair, 
2014). In 1982, the first pilot plant of regenerated cellulose using NMMO as a solvent was built in the United Kingdom (Sinclair 2014). It was later developed into a full-scale commercial process for producing staple cellulose, with "Tencel" as its brand name (Blackburn 2005). Another commercial production process of regenerated cellulose using NMMO as a solvent, known as "lyocell", was established by Lenzing in Austria (Blackburn 2005; Sinclair 2014). In 2004, Lenzing completed the acquisition of Tencel Group and became the largest lyocell fiber producer (Sinclair 2014). In the lyocell process, NMMO with a small amount of water is used as the solvent to produce regenerated cellulose fibers (Chanzy et al. 1990; Bredereck and Hermanutz 2005; Perepelkin 2007).

Apart from the environmental advantage, the lyocell fibers are also of high quality; for example, they have been reported to have high strength and excellent tenacity in the wet state (Loubinoux and Chaunis 1987; Colom and Carrillo 2002; Gindl and Keckes 2006), good adaptability with other fibers, and excellent draping characteristics (Uddin et al. 2010). They also have good thermal resistance and shape stability, which can be used to produce home textile products, sleeping products, a variety of apparel, and industrial products, such as conveyor belts, medical dressings, etc. (Schurz and Lenz 1994; Macfarlane 1997; Burrow 1998).

The objective of this paper is to review the unique advantages of the lyocell process and the desirable properties of lyocell fibers over the closely related, but different, viscose process and viscose fibers. A comparative evaluation of the lyocell and viscose fibers will be given on the basic manufacturing processes, environmental impacts, and product qualities.

\section{MANUFACTURE PROCESSES}

The lyocell manufacturing process (Fig. 1) mainly consists of five steps (Fink et al. 2001; Rosenau et al. 2001; Biganska and Navard 2005; Bredereck and Hermanutz 2005; Hauru et al. 2014):

1) Dissolution: This step includes the disintegration of the pulp fibers and mixing with the solvent. Cellulose is dissolved in an aqueous system containing NMMO to form a dope of high viscosity. The pulp dissolution for the lyocell process is much simpler than that of the viscose process, where the dissolution of dissolving pulp consists of the mercerization stage (steeping with sodium hydroxide), aging, and xanthation using carbon disulfide.

2) Filtration: The formed dope is filtered to remove coarse components. In comparison, the purification of the viscose process includes ripening, filtering, and degassing.

3) Spinning regeneration: The dope is extruded through an orifice spinneret into an air gap, and then regenerated in a coagulation bath. In contrast to this, the production of viscose rayon requires a wet spinning process in an acid bath.

4) Washing: The resulting lyocell fibers are washed and the residual NMMO is recovered and recycled; while the viscose fibers are also washed for removal of the residual $\mathrm{CS}_{2}$, the high volatility of $\mathrm{CS}_{2}$ may lead to its loss via evaporation, causing a negative environmental impact for the viscose process.

5) Finishing: This step involves post-treatment of the fibers, including bleaching, finishing, and drying. 


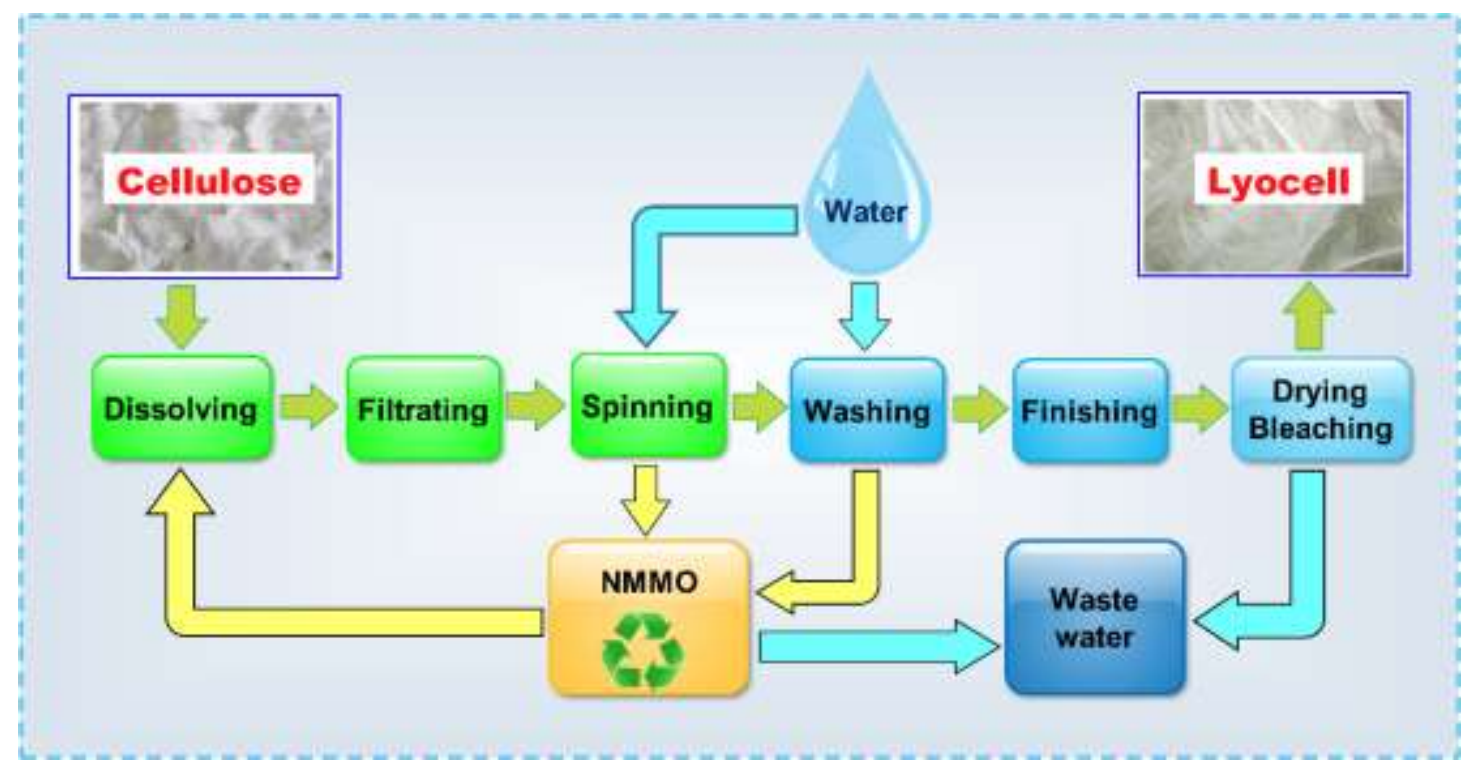

Fig. 1. Manufacture process for the lyocell fibers

The key differences between the lyocell process and the viscose process with respect to dissolution, spinning, and environment are listed in Table 1.

Table 1. Key Differences between the Lyocell Process and the Viscose Processes

\begin{tabular}{|c|c|c|}
\hline Process & Lyocell process/ fibers & Viscose process/ fibers \\
\hline Dissolution & $\begin{array}{l}\text { NMMO direct dissolution } \\
\text { Physical dissolution } \\
\text { Without chemical reactions }\end{array}$ & $\begin{array}{l}\mathrm{NaOH} / \mathbf{C S}_{2} \\
\text { Mercerization/ xanthation } \\
\text { Chemical reaction }\end{array}$ \\
\hline Spinning & $\begin{array}{l}\text { Dry-jet wet spinning } \\
\text { Direct dissolution to form highly viscous } \\
\text { dopes } \\
\text { Fibers are formed via } \\
\text { a) an orifice spinneret in a dry form into } \\
\text { an air gap, } \\
\text { b) then in a coagulation bath. } \\
\text { Irregular molecules arrangement to form } \\
\text { crystallites laminas (Wendler et al. 2011; } \\
\text { Ramamoorthy et al. 2014;). }\end{array}$ & $\begin{array}{l}\text { Wet spinning } \\
\text { Dopes with relatively low viscosity } \\
\text { Fibers are formed using spinneret } \\
\text { immersed in an aqueous bath } \\
\text { containing sulfuric acid and additives. } \\
\text { Stretching is done after the } \\
\text { regeneration. } \\
\text { The formed fibers are relatively } \\
\text { amorphous, with low-tenacity } \\
\text { (Wendler et al. 2011; Ramamoorthy et } \\
\text { al. 2014). }\end{array}$ \\
\hline Environment & $\begin{array}{l}\text { Environmentally friendly } \\
>99 \% \text { of the solvent recovered } \\
\text { No harmful by-products formed } \\
\text { High energy demand for solvent } \\
\text { recycling }\end{array}$ & $\begin{array}{l}\text { Heavy environmental loads } \\
\mathrm{CS}_{2} \text { (about } 25-30 \% \text { ) not recovered } \\
\text { (may also be converted to by-product) } \\
\text { High demand for water }\end{array}$ \\
\hline
\end{tabular}

\section{Solvation and Dissolution}

The mechanism of direct dissolution of cellulose in NMMO (with a small amount of water present), is shown in Fig. 2. Basically, the dissolution process of cellulose includes two stages, i.e., swelling, gradual dissolution. The excellent dissolving capacity of NMMO on cellulose is due to its strong N-O dipoles and basicity, making NMMO an excellent hydrogen bond acceptor solvent. In the dissolution process, the N-O bond, which has a 
stronger basicity than hydroxyl group, will interact with the hydrogen atom on the hydroxyl group of cellulose and form new hydrogen bonds between NMMO and cellulose, replacing the intermolecular and intramolecular hydrogen bonds in cellulose. The amount of bonded NMMO for each glucose unit is critical in dissolving cellulose, and it was found that on average, there were nine NMMO molecules for each glucose unit at the cellulose concentration of 7.5 to $10.5 \%$ (Fink et al. 2001).

A recent study based on all-atom molecular dynamic simulation showed that NMMO could contribute to $75 \%$ to $80 \%$ of the total amount of hydrogen bonds in the NMMO/ cellulose system (Rabideau and Ismail 2015). A thermodynamic simulation study on the crystalline and dissociated states showed that the effect of nonpolar (hydrophobic) effect of NMMO also plays an important role, in addition to the well- known polar (hydrogen bonding) effect (Rabideau and Ismail 2015).

There have been numerous papers documenting the good solubility of wood pulps in NMMO (Fink et al. 2001; Rosenau et al. 2001; Bochek 2003; Schild and Sixta 2011; Ingildeev et al. 2013), such as using the eucalyptus sulfite pulp with a DP of 592 and an $\alpha-$ cellulose content of $94.1 \%$ (Ingildeev et al. 2013), wood pulp having a DP of 750 and $96 \%$ cellulose (Schild and Sixta, 2011), and cotton linters with DP of 759 and $\alpha$-cellulose content of $97.0 \%$ (Ingildeev et al. 2013).

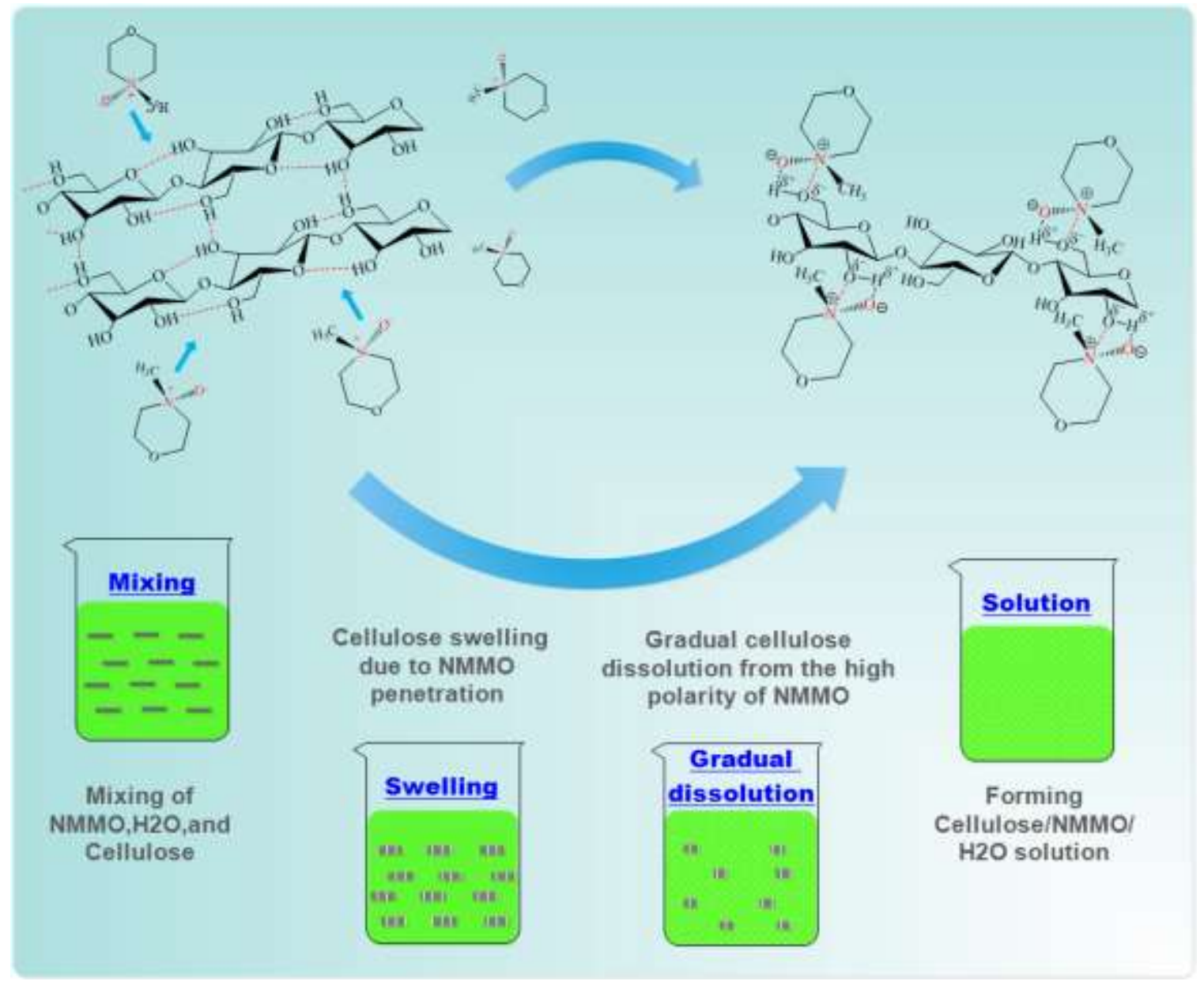

Fig. 2. The mechanism for NMMO dissolution of cellulose (Li et al. 2006; Singha 2012) 
During the dissolution process of cellulose and the recovery / recycling of NMMO (by distillation of NMMO/water solution at high temperatures), a portion of NMMO will be thermally degraded to various compounds due to the homolytic and heterolytic cleavages, which include radical reactions, heterolytic deoxygenation, Polonowski type reaction, and autocatalytic decomposition (Rosenau et al. 2001). The main degradation products were $\mathrm{N}$-methyl morpholine, morpholine, and formaldehyde. When transition metal ions are particularly present in dissolving pulp, the radical reaction of NMMO increases dramatically. To minimize the NMMO degradation, some stabilizers, for example, a combination of alkaline and antioxidant ( $\mathrm{NaOH}$, isopropyl gallate) can be added ( Rosenau et al. 2001; Wendler et al. 2008; Ingildeev et al. 2013), and antioxidants also can be added to the dissolving tank.

During the cellulose pulp dissolution process, both swelling and dissolution occur (Rosenau et al. 2001; Zhang and Tong 2007; Ingildeev et al. 2013). Its first step is to rewet cellulose fibers in a dilute NMMO aqueous solution under the typical conditions of: 50 to $60 \%$ NMMO, 20 to $30 \%$ water, and 10 to $15 \%$ pulp (Rosenau et al. 2001), which will allow great cellulose swelling. Subsequently, an excess amount of water is removed by distilling, such that the desired ratio of cellulose/NMMO/water is obtained for cellulose dissolution Typical compositions are $76 \%$ NMMO, $10 \%$ water, and 14\% cellulose (Rosenau et al. 2001). Under the above conditions, cellulose is dissolved, and a brown, transparent cellulose solution is obtained (Haule et al. 2014). Generally, the ternary solutions are prepared at elevated temperatures from 90 to $120^{\circ} \mathrm{C}$. It is desirable to for more water to be removed to reach the desired ratio of cellulose/NMMO/water to dissolve cellulose to form cellulose dope. However, a high temperature (e.g., higher than $120{ }^{\circ} \mathrm{C}$ ), will cause undesirable loss of NMMO due to degradation (Ingildeev et al. 2013). Therefore, a suitable temperature is desirable.

The direct cellulose dissolution in the NMMO/ water mixture of the lyocell process is fundamentally different from the reaction-dissolution process of the viscose process. Under the strong alkaline conditions of the viscose process, the reactions of carbon disulfide and cellulose result in the formation of cellulose xanthate, which is responsible for the dissolution of cellulose (Bredereck and Hermanutz 2005; Hauru et al. 2014). In addition, the reaction-dissolution of the viscose process requires further steps such as steeping, pressing, shredding and aging, which are time-consuming.

\section{Spinning}

The lyocell process uses the so-called dry jet-wet spinning process, as shown in Fig. 3: 1) the dopes in the NMMO/water system are extruded out from the spinning nozzle, then into an air gap; 2) the formed filament then immediately enters a coagulation bath to continue its formation (Rosenau et al. 2001; Biganska and Navard 2005; Perepelkin 2007). During the above spinning process, the spinning ray and the forming fibers undergo continuous changes. In the viscose process, only a wet spinning is conducted.

In the dry jet-wet spinning process, the spinning process ability depends highly on the process parameters, particularly the dope viscosity, air gap, spinning temperature, and spinning speed. The lyocell dope has a higher viscosity (DP of 500 to 600, based on the Staudinger method) than the viscose dope (DP of 250 to 350, based on the Staudinger method); such a difference is partially responsible for the superior strength properties of the lyocell fiber. Furthermore, the high viscosity of the spinning solution of the lyocell 
process can allow it to maintain a very stable spinning of fibers through the air gap (Haule et al. 2014).

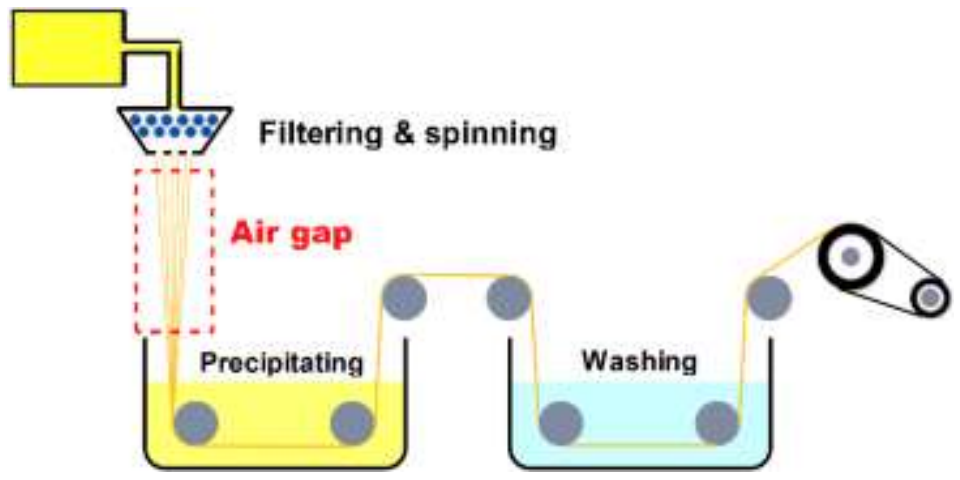

Fig. 3. Dry jet-wet spinning in the lyocell process (Kim et al. 2005)

In fact, the air gap is a critical element of the operation, with significant changes in the solution, and the spinning ray stretched and oriented (Mortimer and Peguy 1996a,b; Mortimer et al. 1996). A highly oriented structure is formed upon entering the aqueous precipitation bath. Air gap length influences the fiber strength (Mortimer and Peguy 1996a). Reducing the air gap length, e.g. from 250 to $20 \mathrm{~mm}$, will reduce the fiber strength. A long air gap causes good orientation, increases in birefringence, and leads to high strength. However, if the air gap is too long, a reduction in orientation occurs, which is caused by relaxation effects before the fixation of the highly oriented solution in the bath. Therefore, an excessively long air gap should also be avoided.

The temperature of the coagulation and the wet spinning speed are also critical factors. When the temperature is fixed, the increase of speed (rise of draw ratio) leads to increases in fiber crystallinity, birefringence, and orientation, which contribute to the improvement in fiber strength (determined by orientation) and initial Young's modulus (determined by crystallinity). However, when the speed is fixed, with the increase of temperature, the fiber structure and properties show changes in the opposite direction (Maron et al. 1994). Good textile properties were reported for a draw ratio of less than 3 and for take-off speeds of $50 \mathrm{~m} / \mathrm{min}$ (Fink et al. 2001).

With the air gap, in addition to the formation of lyocell fiber with highly oriented and crystalline structure, some weaker lateral links between the crystallites can lead to the so-called fibrillation, which is evident as some fibrous elements being exposed at the surface of the lyocell fiber under the condition of wet abrasion (Periyasamy and Khanum 2012). Fibrillation can be reduced via changing the various spinning parameters such as air gap conditions and draw ratio (Mortimer and Peguy 1996a; Periyasamy and Khanum 2012). Usually, a low spinning speed means a long spinning ray in the air gap, which will result in a low fibrillation. On the other hand, with a short air gap, a high draw ratio will increase the fibrillation degree significantly, while a low draw ratio will lead to the production of fibers with good strength but a low fibrillation degree. For long air gaps and draw ratios of up to 10, fibers with very good mechanical properties and low fibrillation can be obtained (Mortimer and Peguy 1996a).

Another key difference is found in the spinning technology: the dry jet-wet spinning (air gap spinning) for the lyocell process, versus the wet spinning (without air gap) for the viscose process. In the latter, there is not enough time for orientation of dope due to lack 
of air gap (Periyasamy and Khanum 2012), which can result in a less orientated fiber structure having lower strength. It should be pointed out that the high orientation of lyocell fibers is unique and contributes significantly to the favorable strength of the material. In fact, this high orientation is only possible with the air gap spinning technology

\section{Environmental Impact}

The environmental advantage of the lyocell process is one of its key features. The direct dissolution technology avoids the use of toxic chemicals such as carbon disulfide; this makes it a more environment-friendly process. More than $99 \%$ of the NMMO solution can be recovered for the lyocell process (Rosenau et al. 2001; Gindl-Altmutter et al. 2014), and NMMO is biodegradable and has no harmful by-products (Woodings 1995; Chen et al. 2015). By contrast, in the viscose process, carbon disulfide is well known for its negative environmental impact, and cost-intensive investment in pollution-control systems must be made for the viscose process (Bredereck and Hermanutz 2005; Hauru et al. 2014). Only 70 to $75 \%$ of the highly volatile and toxic $\mathrm{CS}_{2}$ is recoverable during the viscose fiber production process (Chen et al. 2015).

In addition to the carbon disulfide related issues, the viscose process also uses sodium and zinc salts in the coagulation bath, which also requires the corresponding posttreatments for pollution control (Ingildeev et al. 2013).

The traditional technology to remove water and to recover NMMO in the lyocell process is by distillation. Under the conditions that typically prevail in the industrial process, NMMO may be converted to N-Methyl morpholine and morpholine, etc., which in the literature, is known as "fast exothermic processes" or "thermal runaway reactions" (Brandner and Zengel 1982). Therefore, the chemical stability is a crucial issue for the lyocell process at the commercial scale. Various stabilizers (e.g., propyl gallate), have been studied and shown to be effective (Rosenau et al. 2002). The wash process is critical for an efficient recovery of NMMO. More wash water could potentially wash out more residual NMMO from the fibers (less NMMO loss); however, the energy demand would be too high for the subsequent evaporation. Therefore, it is needed to strike a balance between the amount of water used and the amount of NMMO recovered (Perepelkin 2007; Yau et al. 2013). New technologies such as membrane technologies, electro dialysis, may have potential to remove water from the NMMO/water solution (Perepelkin 2007).

\section{PROPERTIES OF LYOCELL FIBER}

\section{Fiber Structure}

The unique dry jet-wet spinning manufacturing process imparts special features to the structure of the resultant lyocell fibers. Comparison of the lyocell fibers versus viscose fibers is given in Fig. 4. The viscose fibers (Fig. 4a) have quite a rough surface, with oval shaped cross-sections and also more defects (Peng et al. 2003). In contrast, the lyocell fibers (Fig. 4b) show a rather smooth fiber surface, round cross-sections, and homogeneous appearance and dense fibrillar structure (Michud et al. 2015).

The average degree of polymerization and the crystallinity of the lyocell fibers are higher than those of the viscose fibers (Chavan and Patra 2004). The crystallinity index (CrI) of lyocell fibers (0.44) is much higher than that of viscose fibers (0.25) (StanaKleinschek et al. 2001). As a result, the lyocell fibers have higher mechanical properties, 
higher dry and wet tenacity, and higher wet modulus, in comparison with viscose fibers (to be discussed in the next section).

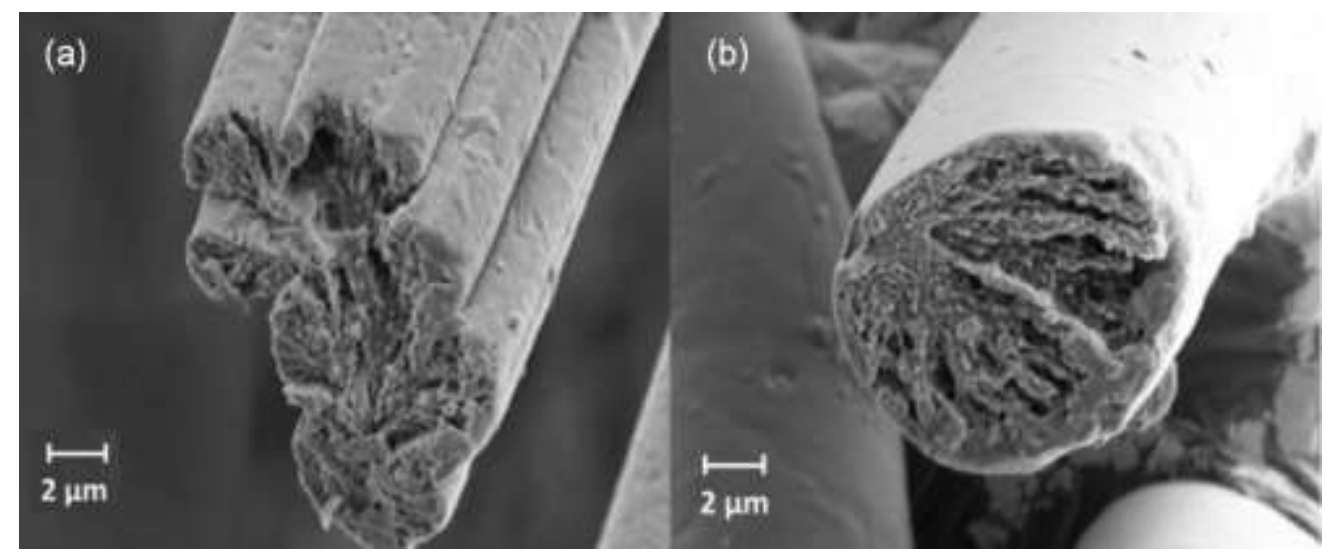

Fig. 4. SEM images of viscose (a); lyocell (b) from eucalyptus prehydrolysis kraft dissolving pulp (Michud et al. 2015)

During the air gap and coagulation stages of the lyocell spinning process, fibers are oriented and then crystallization occurs (Kampl and Leitner 1996; Burrow 2004), forming the unique fiber structure with high crystallinity (Fink et al. 2001), well-oriented amorphous regions, low surface roughness, and longitudinal arrangement (Chanzy et al. 1980). In the same process, some unexpected fibrillation can also occur on the regenerated fiber surface (Fig. 5) (Chavan and Patra 2004; Uddin et al. 2010). Therefore, some actions need to be taken to control the fibrillation. For example, treating lyocell fiber with various types of alkali, dyeing it with poly functional reactive dyes, and using cross-linking agents and enzymatic treatments (Lim et al. 2003; Periyasamy and Khanum 2012).
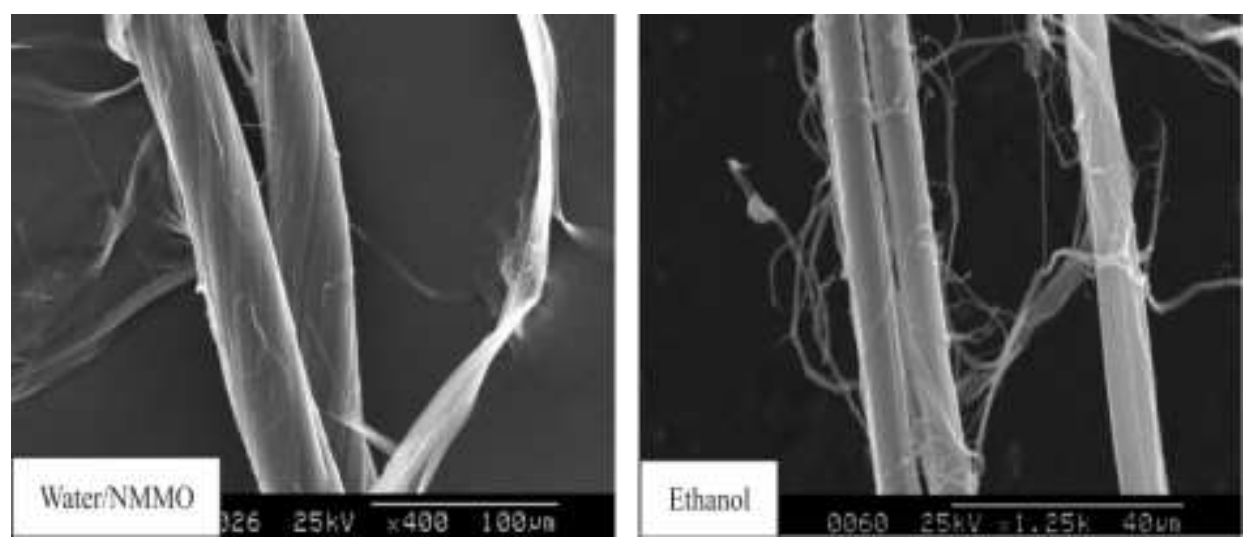

Fig. 5. SEM images of fibrillated bagasse regenerated fibers after coagulation from water/NMMO and ethanol respectively (Uddin et al. 2010).

In contrast, the viscose fibers have lower crystallinity and lower fibril orientation. Besides, compared to the lyocell fibers, the viscose fibers have a less homogeneous appearance (Fig. 4), and they also show a distinct "skin-core" structure (Fig. 6) (Abu-Rous et al. 2006). The orientation degree and density in the skin-region are higher than those in the fiber core (Biganska et al. 2002; Eichhorn et al. 2003; Abu-Rous et al. 2006). The 
formation of "skin-core" structure in the viscose fiber is due to the heterogeneous precipitations taking place on the fiber cross-section during the wet spinning process: the viscose dope is extruded to the acid bath, and the a regenerated "skin" layer is formed first, then sulfuric acid gradually diffuses through the "skin" and solidifies the interior (Hauru et al. 2014).

Cross-linking is commonly practiced in the industry, which effectively reduces fibrils on the fiber surface (Bredereck et al. 1997). The lyocell fibers are more challenging in this regard in comparison to the viscose fibers in fabric and garment (Bates et al. 2004). Cross-linking agents, such as 2,4-diacryl- amido- benzene sulphonic acid have been known for many years to treat lyocell fibers, and they are typically small molecules containing multiple functional groups, (e.g., they are capable of reacting with the hydroxy groups in cellulose to form crosslinks (Potter and Taylor 1998)).

There are big differences in the use of additives between the lyocell process and the viscose process. In the case of the lyocell process, the main objective is to increase the chemical stability of NMMO. Stabilizers (e.g., propyl gallate, ) have been demonstrated to be effective (Rosenau et al. 2005). Wendler et al. (2005) reported the use of a polymeric stabilizer system consisting of imino- diacetic acid sodium salt (ISDB) and benzyl amine (BSDB) covalently bound to a styrene/divinyl benzene copolymer in the cellulose/NMMO solutions. Such a system shows distinct improvements in the chemical stability of the Lyocell solution compared to the $\mathrm{NaOH} /$ propyl gallate stabilizer system (Wendler et al. 2006).

Additives are also applied in the viscose process, for example to control the rates of coagulation and regeneration and to reduce primary swelling of gel fibers (Levine and Burroughs 1959). These additives include aliphatic amines, dithio- carbamates and oxide derivatives of amine (Arthur 1948; Louis 1950; Maclaurin and Israel 1952). Recent development also includes the addition of antibacterial compounds to the spinning solution to impart antibacterial properties to the fibers (Bredereck and Hermanutz 2005a).

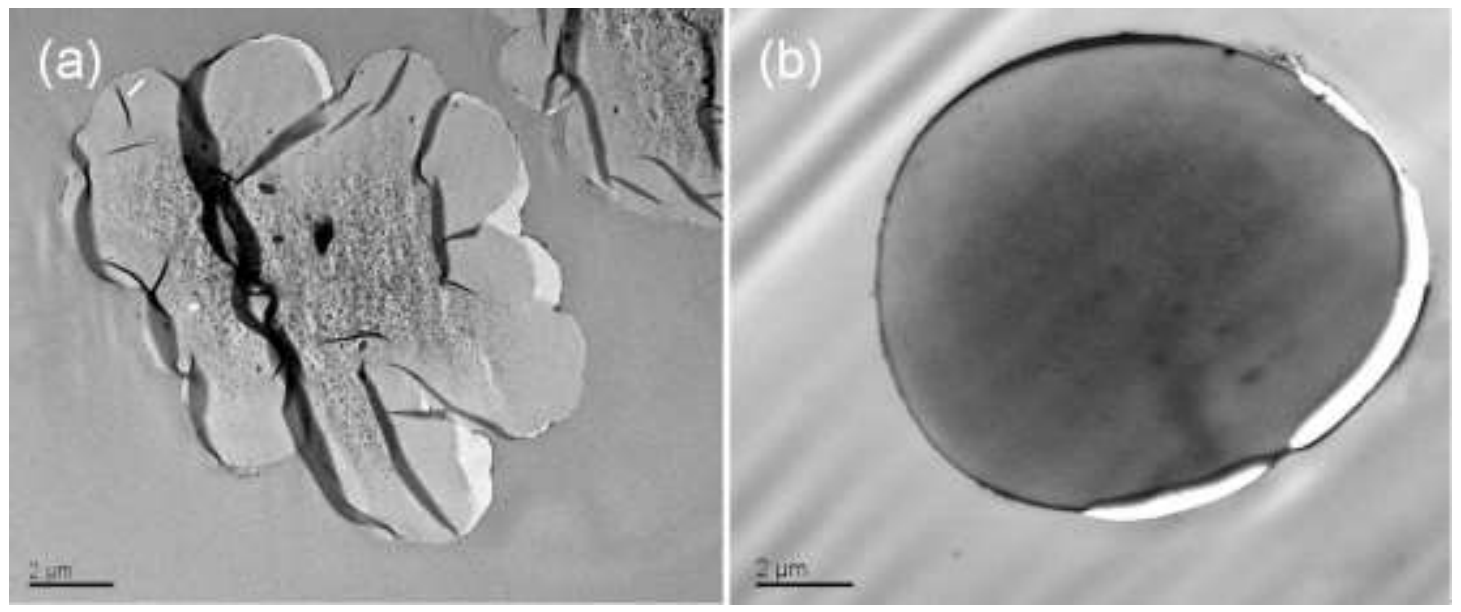

Fig. 6. TEM images of cross sections: Viscose fiber (a); lyocell fiber (b) (Abu-Rous et al. 2006).

\section{Mechanical Properties}

Typical mechanical properties of lyocell fibers, in comparison with viscose fibers, are shown in Table 2 (Perepelkin 2007). 
Table 2. Typical Mechanical Properties of Lyocell Fibers Comparing with Viscose Fibers

\begin{tabular}{|c|c|c|c|}
\hline Properties & & Lyocell & Viscose \\
\hline Tenacity (cN/dtex) & dry & $40-42$ & $22-26$ \\
wet & $34-38$ & $10-15$ \\
\hline Deformation modulus & dry & $8-10$ & $3-5$ \\
(GPa) & wet & $3-4.5$ & $0.6-1$ \\
\hline Preservation of strength & loop & $30-40$ & $30-40$ \\
$(\%)$ & wet & $60-80$ & $50-55$ \\
\hline Loop strength (cN/tex) & -- & 19 & 6 \\
\hline Elongation (\%) & Dry & $11-16$ & $17-25$ \\
& wet & $16-18$ & $21-30$ \\
\hline
\end{tabular}

The lyocell fiber has excellent strength properties, in both dry and wet states. As shown in Table 2, the lyocell fiber has very high dry tenacity and modulus. The dry tensile strength is higher than the viscose fiber, and it retains much of its strength when wet, while the viscose fiber loses about half of its tensile strength when wet. The data in Table 2, also show that compared to viscose, the lyocell fiber is twice stronger when dry and three times when wet. The reasons include the higher orientation of the lyocell fiber (Chavan and Patra 2004). Also, compared to viscose fiber, the lyocell fiber is more than two times higher in modulus and almost five times when wet (Krüger 1994; Chavan and Patra 2004; Weigel et al. 1994). As noted in Table 2, the lyocell fiber has a lower elongation at break, which may be related to the higher crystalline zone and uniform structures in contrast to viscose fiber.

Besides, other properties of the lyocell fiber, such as softness, absorbency, breathability, thermal resistance and shape stability, dyeability, and luxurious handle of lyocell fiber, are superior to those of the viscose fiber (Burrow 2004; Borbély 2008; Uddin et al. 2010).

It should be noted that in addition to regular rayon fibers, there are some major modifications of rayon fibers. The regular rayon has the largest market share, and its major application is for apparel and home furnishings, with the term "viscose" on their commercial labels. Regular rayon has a low wet strength. As a result, it becomes unstable and may stretch or shrink when wet. Special rayon fibers, such as Modal or polynosic fibers, can have much improved mechanical properties (Röder et al. 2009). For example, Modal is a trade name for special viscose fibers that have high wet modulus (HWM) (Lenz et al. 1988). In fact, Modal fibers have very similar properties to regular rayon, other than their high wet strength. Modal rayons can be machine washed and tumble dried and perform much like cotton in similar end uses. (Cook 1984).

\section{APPLICATIONS OF LYOCELL FIBERS}

Due to their inherent properties, lyocell fibers have been widely used in many applications, including textiles and various consumer and industrial products.

Lyocell fibers have the characteristics of high strength, soft and silky handling, luster and bulky touch, biodegradability, high absorbency, and easy processing (Borbély 2008), which could then be suitable for nonwovens. Lyocell fibers are good raw materials for house textile products, including mattresses, mattress pads, bed covers, linens (Macfarlane 1997; Burrow 1998); for different apparels, e. g., denim, chino, underwear, 
casual wear, and towels. Furthermore, filament lyocell fibers are excellent for both men's and women's clothing (Macfarlane 1997; Burrow 1998).

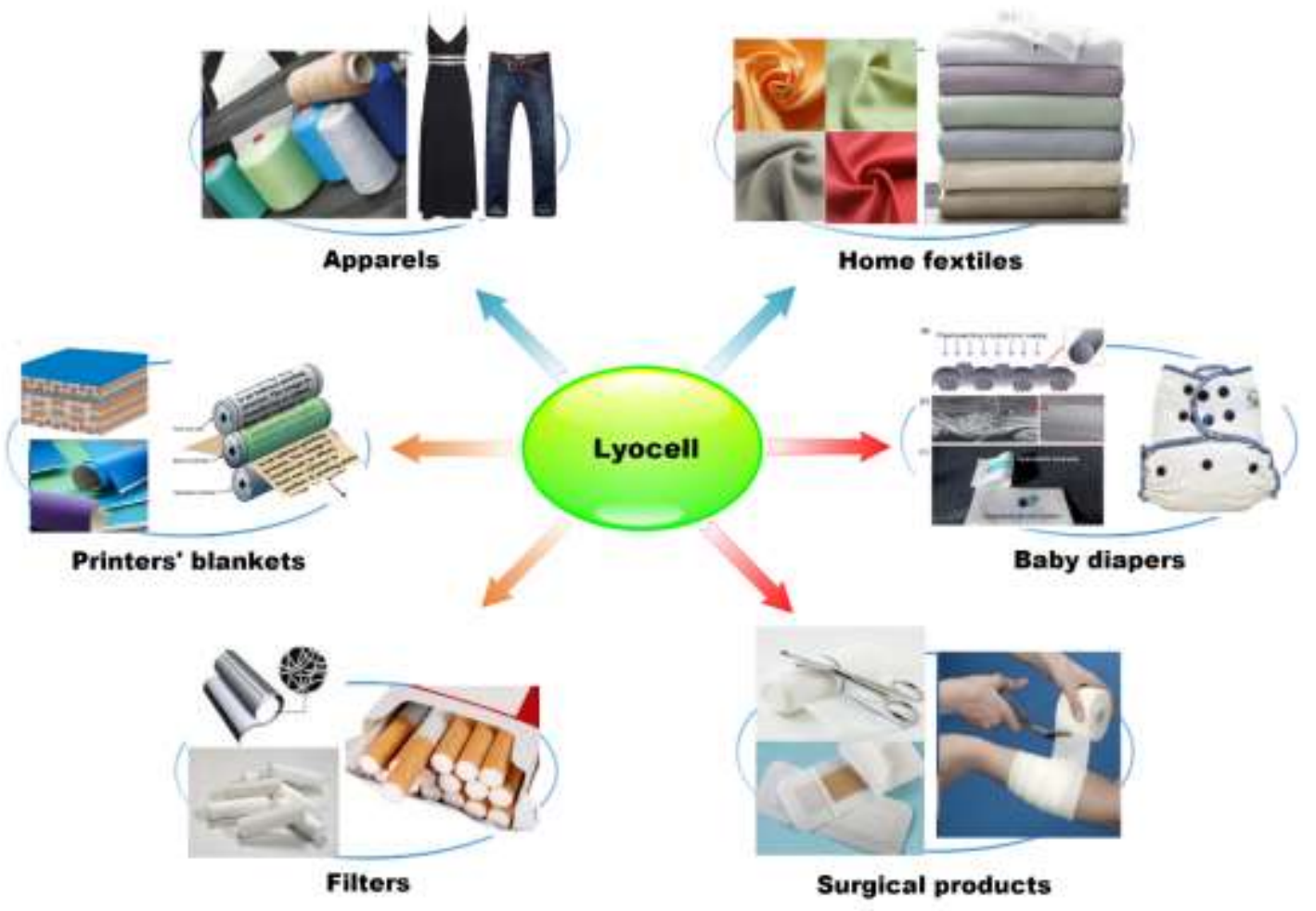

Fig. 7. Various applications of lyocell fibers

The non-textile products made from lyocell fibers include wipes, medical swabs, gauzes, filters, biocomposites, and battery separators (Chavan Patra 2004; Wang et al., 2011). In addition, lyocell fibers were used to produce conveyor belts due to the high modulus and shape stability. Specialty paper can be produced from lyocell fibers, which can have excellent strength, opacity, and low air resistance (Borbély 2008).

However, the lyocell process is still in its early stage of commercial development in comparison with the well-established viscose process, and one of the most important factors in hindering the rapid and wide adoption is its high cost (Wang et al. 2012). Therefore, it is desirable to decrease the overall production cost of lyocell fibers; for example, by using more cost-effective sources of pulps as the raw materials. It was reported that using high hemicellulose content pulp can be a good way to decrease the production cost for lyocell fibers (Zhang and Tong 2007; Zhang et al. 2008).

\section{CONCLUSIONS}

The NMMO-based lyocell process and its unique production processes, such as dry jet-wet spinning, have received many commercial interests. In contrast to the commercial viscose fiber manufacturing process, the NMMO-based lyocell process is much more environmentally friendly and efficient. The overall manufacturing process for lyocell fiber includes dissolution, spinning (deformation, cooling, relaxation, coagulation), washing and 
finishing. Lyocell fibers have unique fiber structure and properties, and they have rather a uniform fibril orientation between the central and the periphery region of the fibers, high crystallinity, long crystallites, thus possessing very high strength and modulus in comparison with viscose fibers. These favorable properties render lyocell fibers suitable for many applications. With the continuing effort in decreasing the overall production cost, the lyocell process/ fibers can be expected to have increased importance in the production of regenerated cellulose products in the future.

\section{REFERENCES CITED}

Abu-Rous, M., Ingolic, E., and Schuster, K. (2006). "Visualisation of the nano-structure of Tencel® (lyocell) and other cellulosics as an approach to explaining functional and wellness properties in textiles," Lenzinger Berichte 85, 31-37.

Biganska, O., and Navard, P. (2005). "Kinetics of precipitation of cellulose from cellulose-NMMO-water solutions," Biomacromolecules 6(4), 1948-1953. DOI: $10.1021 / \mathrm{bm} 040079 \mathrm{q}$

Biganska, O., Navard, P., and Bédué, O. (2002). "Crystallisation of cellulose/Nmethylmorpholine-N-oxide hydrate solutions," Polymer 43(23), 6139-6145. DOI: 10.1016/S0032-3861(02)00552-9

Blackburn, R. S. (2005). Biodegradable and Sustainable Fibres (Vol. 47)., Taylor \& Francis US.

Bochek, A. (2003). "Effect of hydrogen bonding on cellulose solubility in aqueous and nonaqueous solvents," Russian Journal of Applied Chemistry 76(11), 1711-1719. DOI: 10.1023/B:RJAC.0000018669.88546.56

Borbély, É. (2008). "Lyocell, the new generation of regenerated cellulose," Acta Polytechnica Hungarica 5(3), 11-18.

Brandner, A., and Zengel, H. (1982). "Molding and spinning compositions based on cellulose with a low content of low-molecular-weight degradation products," Paper presented at the Chem Abstr.

Bredereck, K., and Hermanutz, F. (2005). "Man-made cellulosics," Review of Progress in Coloration and Related Topics 35(1), 59-75. DOI: 10.1111/j.14784408.2005.tb00160.x

Burrow, T. (1998). "Recent results with lyocell fibers in textiles," Lenz Ber 78, 37-40.

Burrow, T. (2004). "Recent advances in chemically treated lyocell fibers," Chemical Fibers International 54, 304-305.

Chanzy, H., Paillet, M., and Hagege, R. (1990). "Spinning of cellulose from N-methyl morpholine N-oxide in the presence of additives," Polymer 31(3), 400-405. DOI: 10.1016/0032-3861(90)90376-A

Chanzy, H., Peguy, A., Chaunis, S., and Monzie, P. (1980). “Oriented cellulose films and fibers from a mesophase system," Journal of Polymer Science: Polymer Physics Edition 18(5), 1137-1144. DOI: 10.1002/pol.1980.180180517

Chavan, R., and Patra, A. (2004). "Development and processing of lyocell," Indian Journal of Fibre and Textile Research 29(4), 483.

Chen, C., Duan, C., Li, J., Liu, Y., Ma, X., Zheng, L., Stavik, J., and Ni, Y. (2016). "Cellulose (dissolving pulp) manufacturing processes and properties: A mini review," BioResources 11(2), 5553-5564. 
Chen, J.-H., Guan, Y., Wang, K., Xu, F., and Sun, R.-C. (2015). "Regulating effect of hemicelluloses on the preparation and properties of composite lyocell fibers," Cellulose 22(3), 1505-1516. DOI: 10.1007/s10570-015-0608-0

Colom, X., and Carrillo, F. (2002). "Crystallinity changes in lyocell and viscose-type fibres by caustic treatment," European Polymer Journal 38(11), 2225-2230. DOI: 10.1016/S0014-3057(02)00132-5

Duan, C., Li, J., Ma, X., Chen, C., Liu, Y., Stavik, J., and Ni, Y. (2015). "Comparison of acid sulfite (AS)-and prehydrolysis kraft (PHK)-based dissolving pulps," Cellulose 22(6), 4017-4026. DOI: 10.1007/s10570-015-0781-1

Duan, C., Verma, S. K., Li, J., Ma, X., and Ni, Y. (2016). "Combination of mechanical, alkaline and enzymatic treatments to upgrade paper-grade pulp to dissolving pulp with high reactivity," Bioresource Technology 200, 458-463. DOI: 10.1016/j.biortech.2015.10.067

Eichhorn, S., Young, R., Davies, R., and Riekel, C. (2003). "Characterisation of the microstructure and deformation of high modulus cellulose fibres," Polymer 44(19), 5901-5908. DOI: 10.1016/S0032-3861(03)00540-8

Fink, H.-P., Weigel, P., Purz, H., and Ganster, J. (2001). "Structure formation of regenerated cellulose materials from NMMO-solutions," Progress in Polymer Science 26(9), 1473-1524. DOI: 10.1016/S0079-6700(01)00025-9

Gindl-Altmutter, W., Eichhorn, S. J., Burghammer, M., and Keckes, J. (2014). "Radial crystalline texture in a lyocell fibre revealed by synchrotron nanofocus wide-angle X-ray scattering," Cellulose 21(1), 845-851. DOI: 10.1007/s 10570-013-0140-z

Gindl, W., and Keckes, J. (2006). "Strain hardening in regenerated cellulose fibres," Composites Science and Technology 66(13), 2049-2053. DOI:

10.1016/j.compscitech.2005.12.019

Haemmerle, F. M. (2011). "The cellulose gap," Lenzing Ber 89, 12-21.

Haule, L., Carr, C., and Rigout, M. (2014). "Investigation into the removal of an easycare crosslinking agent from cotton and the subsequent regeneration of lyocell-type fibres," Cellulose 21(3), 2147-2156. DOI: 10.1007/s10570-014-0225-3

Hauru, L. K., Hummel, M., Michud, A., and Sixta, H. (2014). "Dry jet-wet spinning of strong cellulose filaments from ionic liquid solution," Cellulose 21(6), 4471-4481. DOI: $10.1007 / \mathrm{s} 10570-014-0414-0$

Ingildeev, D., Effenberger, F., Bredereck, K., and Hermanutz, F. (2013). "Comparison of direct solvents for regenerated cellulosic fibers via the lyocell process and by means of ionic liquids," Journal of Applied Polymer Science 128(6), 4141-4150. DOI: 10.1002/app.38470

Kampl, R., and Leitner, H. (1996). "Processing behavior and applications of lyocell," Lenzinger Berichte (75), 97.

Kim, D. B., Pak, J. J., Jo, S. M., and Lee, W. S. (2005). "Dry jet-wet spinning of cellulose/N-methylmorpholine N-oxide hydrate solutions and physical properties of lyocell fibers," Textile Research Journal 75(4), 331-341. DOI: 10.1177/0040517505054852

Krüger, R. (1994). “Cellulosic filament yarn from the NMMO process," Lenz Ver 74, 49. Li, H.-J., Cao, Y.-M., Qin, J.-J., Jie, X.-M., Wang, T.-H., Liu, J.-H., and Yuan, Q. (2006). "Development and characterization of anti-fouling cellulose hollow fiber UF membranes for oil-water separation," Journal of Membrane Science 279(1), 328335. DOI: 10.1016/j.memsci.2005.12.025 
Li, J., Zhang, H., Duan, C., Liu, Y., and Ni, Y. (2015). "Enhancing hemicelluloses removal from a softwood sulfite pulp," Bioresource Technology 192, 11-16. DOI: 10.1016/j.biortech.2015.04.107

Lim, K. Y., Seong, Y. J., and Kim, B. C. (2003). "Reduction of fibrillation of lyocell fiber with cellulose-g-poly (vinyl alcohol) copolymer,” Polymer Journal 35(9), 691-696. DOI: 10.1295/polymj.35.691

Loubinoux, D., and Chaunis, S. (1987). "An experimental approach to spinning new cellulose fibers with N-methylmorpholine-oxide as a solvent," Textile Research Journal 57(2), 61-65. DOI: 10.1177/004051758705700201

Macfarlane, K. (1997). "Nonwovens applications of lyocell fibers," Chemical Fibers International 47(4), 328-332.

Maron, R., Michels, C., and Taeger, E. (1994). "Investigations for preparation of cellulose solutions in NMMO and the following forming," Lenzinger Berichte 74(9), 27-29.

Miao, Q., Chen, L., Huang, L., Tian, C., Zheng, L., and Ni, Y. (2014). “A process for enhancing the accessibility and reactivity of hardwood kraft-based dissolving pulp for viscose rayon production by cellulase treatment," Bioresource Technology 154, 109-113. DOI: 10.1016/j.biortech.2013.12.040

Michud, A., Tanttu, M., Asaadi, S., Ma, Y., Netti, E., Kääriainen, P., Persson, A., Berntsson, A., Hummel, M. and Sixta, H. (2015). "Ioncell-F: ionic liquid-based cellulosic textile fibers as an alternative to viscose and lyocell," Textile Research Journal 86(5), 543-552. DOI: 10.1177/0040517515591774

Mortimer, S., and Peguy, A. (1996a). "The formation of structure in the spinning and coagulation of lyocell fibres," Cellulose Chemistry and Technology 30(1-2), 117132.

Mortimer, S., and Peguy, A. (1996b). "The influence of air-gap conditions on the structure formation of lyocell fibers," Journal of Applied Polymer Science 60(10), 1747-1756. DOI: 10.1002/(SICI)1097-4628(19960606)60:10<1747::AIDAPP28>3.0.CO;2-\#

Mortimer, S., Peguy, A., and Ball, R. (1996). "Influence of the physical process parameters on the structure formation of lyocell fibres," Cellulose Chemistry and Technology 30(3-4), 251-266.

Perepelkin, K. (2007). "Lyocell fibres based on direct dissolution of cellulose in Nmethylmorpholine N-oxide: Development and prospects," Fibre Chemistry 39(2), 163-172. DOI: 10.1007/s10692-007-0032-9

Periyasamy, A. P., and Khanum, M. R. (2012). "Effect of fibrillation on pilling tendency of lyocell fiber," Bangladesh Textile Today 4, 31-39.

Quintana, E., Valls, C., Vidal, T., and Roncero, M. B. (2013). “An enzyme-catalysed bleaching treatment to meet dissolving pulp characteristics for cellulose derivatives applications," Bioresource Technology 148, 1-8. DOI:

10.1016/j.biortech.2013.08.104

Rabideau, B. D., and Ismail, A. E. (2015). "Effect of water content in N-methylmorpholine N-oxide/cellulose solutions on thermodynamics, structure, and hydrogen bonding," The Journal of Physical Chemistry B 119(48), 15014-15022. DOI: 10.1021/acs.jpcb.5b07500 
Ramamoorthy, S. K., Skrifvars, M., and Rissanen, M. (2014). "Effect of alkali and silane surface treatments on regenerated cellulose fibre type (lyocell) intended for composites," Cellulose 22(1), 637-654. DOI: 10.1007/s10570-014-0526-6

Rosenau, T., Potthast, A., Sixta, H., and Kosma, P. (2001). "The chemistry of side reactions and byproduct formation in the system NMMO/cellulose (lyocell process)," Progress in Polymer Science 26(9), 1763-1837. DOI: 10.1016/S00796700(01)00023-5

Schild, G., and Sixta, H. (2011). "Sulfur-free dissolving pulps and their application for viscose and lyocell," Cellulose 18(4), 1113-1128. DOI: 10.1007/s10570-011-9532-0

Schurz, J., and Lenz, J. (1994). "Investigations on the structure of regenerated cellulose fibers; Herrn Professor Janeschitz-Kriegl zum 70. Geburtstag mit den besten Wünschen gewidmet," in Macromolecular symposia (Vol. 83, No. 1, pp. 273-289). Hüthig \& Wepf Verlag.

Sinclair, R. (2014). Textiles and Fashion: Materials, Design and Technology, Elsevier. Singha, K. (2012). "Importance of the phase diagram in lyocell fiber spinning," Int. J. Mater. Eng. 2(3), 10-16.

Sixta, H., Iakovlev, M., Testova, L., Roselli, A., Hummel, M., Borrega, M., van Heiningen, A., Froschauer, C. and Schottenberger, H. (2013). "Novel concepts of dissolving pulp production," Cellulose 20(4), 1547-1561. DOI: 10.1007/s10570013-9943-1

Tian, C., Zheng, L., Miao, Q., Cao, C., and Ni, Y. (2014). "Improving the reactivity of kraft-based dissolving pulp for viscose rayon production by mechanical treatments," Cellulose 21(5), 3647-3654. DOI: 10.1007/s10570-014-0332-1

Uddin, A. J., Yamamoto, A., Gotoh, Y., Nagura, M., and Iwata, M. (2010). "Preparation and physical properties of regenerated cellulose fibres from sugarcane bagasse," Textile Research Journal 80(17), 1846-1858. DOI: 10.1177/0040517510369408

Wang, H., Gurau, G., and Rogers, R. D. (2012). "Ionic liquid processing of cellulose," Chemical Society Reviews 41(4), 1519-1537. DOI: 10.1039/C2CS15311D

Wang, L., Zhang, Y., Gao, P., Shi, D., Liu, H., and Gao, H. (2006). "Changes in the structural properties and rate of hydrolysis of cotton fibers during extended enzymatic hydrolysis," Biotechnology and Bioengineering 93(3), 443-456. DOI: 10.1002/bit.20730

Wang, Q., Liu, S., Yang, G., Chen, J., and Ni, Y. (2015). “Cationic polyacrylamide enhancing cellulase treatment efficiency of hardwood kraft-based dissolving pulp," Bioresource Technology 183, 42-46. DOI: 10.1016/j.biortech.2015.02.011

Wang X., Jian, H., and Jin, L. (2011). "Preparation ultra-fine fibrillated lyocell fiber and its application in battery separator," Int. J. Electrochem. Sci 6, 4999-5004.

Weigel, P., Gensrich, J., and Fink, H. (1994). "The structural format of cellulose fibers from amine oxide solutions," Lenz Ber 74, 31-37.

Wendler, F., Konkin, A., and Heinze, T. (2008). "Studies on the stabilization of modified lyocell solutions," in: Macromolecular Symposia (Vol. 262, No. 1, pp. 72-84). Wiley-VCH Verlag. DOI: 10.1002/masy.200850208

Wendler, F., Persin, Z., Stana-Kleinschek, K., Reischl, M., Ribitsch, V., Bohn, A., Fink, H.P. and Meister, F. (2011). "Morphology of polysaccharide blend fibers shaped from $\mathrm{NaOH}, \mathrm{N}$-methylmorpholine-N-oxide and 1-ethyl-3-methylimidazolium acetate," Cellulose 18(5), 1165-1178. DOI: 10.1007/s10570-011-9559-2 
Woodings, C. (1995). "The development of advanced cellulosic fibres," International Journal of Biological Macromolecules 17(6), 305-309. DOI: 10.1016/01418130(96)81836-8

Yau, E., Badal, K., Brodeur, G., Collier, J., Telotte, J., and Ramakrishnan, S. (2013). "Enzymatic hydrolysis of N-methyl morpholine n-oxide and ionic liquid-treated cellulose: A comparative study," Indian Chemical Engineer 55(4), 235-246. DOI: 10.1080/00194506.2013.851993

Zhang, H., and Tong, M. (2007). "Influence of hemicelluloses on the structure and properties of lyocell fibers," Polymer Engineering \& Science 47(5), 702-706. DOI: 10.1002/pen.20743

Zhang, H., Zhang, H., Tong, M., Shao, H., and Hu, X. (2008). "Comparison of the structures and properties of lyocell fibers from high hemicellulose pulp and high $\alpha$ cellulose pulp," Journal of Applied Polymer Science 107(1), 636-641. DOI: 10.1002/app.27129

Article submitted: January 10, 2018; Peer review completed: February 17, 2018; Revised version received: February 26, 2018; Accepted: February 28, 2018; Published: March 13, 2018.

DOI: 10.15376/biores.13.2.Zhang 